\title{
Fast Multi-Symbol Based Iterative Detectors for UWB Communications
}

\author{
Qi Zhou, ${ }^{1}$ Xiaoli Ma, ${ }^{1}$ and Vincenzo Lottici ${ }^{2}$ \\ ${ }^{1}$ School of Electrical and Computer Engineering, Georgia Institute of Technology, Atlanta GA 30332, USA \\ ${ }^{2}$ Department of Information Engineering, University of Pisa, 56122 Pisa, Italy
}

Correspondence should be addressed to Qi Zhou, qzhou32@gatech.edu

Received 1 November 2009; Accepted 20 May 2010

Academic Editor: Tim Davidson

Copyright (C) 2010 Qi Zhou et al. This is an open access article distributed under the Creative Commons Attribution License, which permits unrestricted use, distribution, and reproduction in any medium, provided the original work is properly cited.

\begin{abstract}
Ultra-wideband (UWB) impulse radios have shown great potential in wireless local area networks for localization, coexistence with other services, and low probability of interception and detection. However, low transmission power and high multipath effect make the detection of UWB signals challenging. Recently, multi-symbol based detection has caught attention for UWB communications because it provides good performance and does not require explicit channel estimation. Most of the existing multi-symbol based methods incur a higher computational cost than can be afforded in the envisioned UWB systems. In this paper, we propose an iterative multi-symbol based method that has low complexity and provides near optimal performance. Our method uses only one initial symbol to start and applies a decision directed approach to iteratively update a filter template and information symbols. Simulations show that our method converges in only a few iterations (less than 5), and that when the number of symbols increases, the performance of our method approaches that of the ideal Rake receiver.
\end{abstract}

\section{Introduction}

Ultra-wideband (UWB) impulse radio (IR) transmits ultrashort pulses at low power spectral density where the information is encoded via pulse-amplitude modulation (PAM) or via pulse-position modulation (PPM). The IR-UWB systems show some important merits including: coexistence with current narrowband signals, high multiple-access capacity and fine timing resolution [1-3]. Fine timing resolution property helps the receiver to resolve distinct dense multipath components and provides high degrees of diversity whilst the low power spectral density enables sharing of the RF spectrum with limited mutual interference.

One of the major challenges in UWB system is to deal with the dense multipath channel. Indeed, each transmitted UWB pulse arrives at the receiver as hundreds of replicas with different delays, amplitudes and phases [4-6]. To collect the available diversity, Rake receivers $[7,8]$ employ a large number of fingers to capture the multipath energy [9]. However, channel estimation error can degrade the Rake's performance and the accurate estimation of the gains and delays of channel paths incurs considerable computational cost [10].

As an alternative to the Rake receiver, the transmitted reference (TR) method $[8,11-14]$ sends a reference signal along with the data-modulated signal. The receiver can simply be an autocorrelation receiver which demodulates the data by correlating the delayed reference signal and the data-modulated signal. The advantage of the TR method compared to the Rake method is that it is easier to implement because it does not require explicit channel estimation. However, the main drawback of TR-based methods is that the noise induced in the reference signal severely degrades the error performance.

In [15], decision-directed autocorrelation (DDA) receivers are proposed to detect the current symbol by correlating the current information waveform with a waveform template generated by all previously decoded symbols. However, the DDA receivers detect the information symbols successively and the current detected symbol has no contribution to the preceding symbol detection. To relieve the noise effect of the reference signal in TR 
system, further enhancement techniques exploit the multisymbol differential detection $[16,17]$ to jointly detect $M$ consecutive symbols. The generalized likelihood ratio test (GLRT) approach for the multi-symbol case is derived and exhaustive search is performed on all $2^{M}$ symbol possibilities to find the optimal one [16]. The practical implementation of the method suffers from the exponential computational complexity in terms of block size $M$. A reduced complexity algorithm is devised in [17] by introducing the sphere decoding algorithm (SDA). An approximate algorithm based on the Viterbi algorithm (VA) is also presented in [17]. Although SDA and VA reduce the complexity relative to exhaustive search, and are effective for small $M$, they require considerable computational effort when $M$ is large.

In this paper, we propose a fast multi-symbol iterative detection method. The method harvests the benefits from the concept of the multiple symbols detection and outputs a better bit error rate (BER) performance than the single symbol TR system whilst exhibiting a low computational complexity $\left(\mathcal{O}\left(N M^{2}\right)\right.$, where $M$ is block size and $N$ is the maximum number of iterations). Following the description of general iterative method, two particular low-complexity detectors are designed and evaluated in the simulation experiments. Although the proposed method cannot guarantee to achieve the same performance as the GLRT-based detector in the general case, experimental results show that the BER performance of the method is very close to that of the GLRT when $M \geq 10$ (the signal-to-noise ratio (SNR) gap is less than $0.5 \mathrm{~dB}$ ). Further experiments demonstrate that a few iterations $(N<5$ iterations $)$ are sufficient for the detectors to converge.

The rest of the paper is organized as follows. Section 2 introduces the UWB signal model. Section 3 describes the multi-symbol transmitted reference system with GLRT detection. Section 4 develops two fast multi-symbol transmitted reference-based detectors. Section 5 shows the numerical results for a constant channel and random channels, respectively. Section 6 concludes the paper.

\section{Signal Model}

The transmitted signal in IR-UWB systems using the pulse amplitude modulation (PAM) for the $i$ th transmitted symbol is

$$
s_{i}(t)=b_{i} \sum_{j=0}^{N_{f}-1} p\left(t-j T_{f}-c_{j} T_{c}\right),
$$

where $p(t)$ is a transmitted monocycle waveform with support set $\left[0, T_{p}\right]$, the $b_{i}$ 's are the modulated symbols, the $c_{j}$ 's are the user-specific pseudorandom time-hopping $(\mathrm{TH})$ codes and $T_{f}$ is its frame duration. Because the energy of one single pulse is limited in UWB communication systems, each symbol is transmitted using $N_{f}$ frames so that the receiver can collect enough energy to recover the signal. Thus, the symbol duration is $T_{s}=N_{f} T_{f}$. The TH codes $c_{j}$ are integers chosen from $0 \leq c_{j} \leq N_{c}-1$ so that multiple users can access the channel concurrently and the transmission time of $j$ th monocycle waveform is delayed with $c_{j} T_{c}$ seconds. Due to the highly-frequency selective feature of UWB channel, the frame duration is chosen such that $T_{f}>T_{m}+T_{p}+$ $N_{c} T_{c}$, where $T_{m}$ is the maximum excess delay of the channel. This condition eliminates intersymbol interference (ISI). The energy of one pulse is $E_{p}=\int_{0}^{T_{p}} p^{2}(t) d t$.

The channel impulse response (CIR) of the UWB system is assumed to be slow fading with multipath propagation

$$
h(t)=\sum_{k=1}^{K} \alpha_{k} \delta\left(t-\tau_{k}\right),
$$

where $K$ is the total number of specular propagation paths with amplitude $\alpha_{k}$ and delay $\tau_{k}$. Hence, the signal obtained from the receiver side for the $i$ th symbol is modeled as

$$
\begin{aligned}
r_{i}(t) & =s_{i}(t) * h(t)+n(t) \\
& =\sum_{k=1}^{K} \alpha_{k} s_{i}\left(t-\tau_{k}\right)+n(t) \\
& =b_{i} \sum_{j=0}^{N_{f}-1} g\left(t-j T_{f}-c_{j} T_{c}\right)+n(t),
\end{aligned}
$$

where $g(t)=p(t) * h(t)$ is the channel template, * denotes the convolution operation and $n(t)$ denotes the noise including multiple access interference (MAI) and an additive white Gaussian noise (AWGN) with zero mean and twosided power spectral density $N_{0} / 2$. The noiseless received signal energy in each frame is defined as $E_{f}=\int_{0}^{T_{f}} g^{2}(t) d t$ and is proportional to the pulse energy $E_{p}$.

A key element to determine the receiver demodulation structure is the way to encode the information symbols $a_{k} \in$ $\{+1,-1\}$ to the transmitted symbols $b_{j} \in\{+1,-1\}$. In the following, we list three kinds of encoders:

(i) Transmitted Reference (TR) [12] with $b_{m}=1$ if $m$ is even, otherwise $b_{m}=a_{(m-1) / 2}$.

(ii) Multi-Symbol Differential Encoder (MSDE) [17] with $b_{i(M+1)}=1$ and $b_{i(M+1)+m}=b_{i(M+1)+m-1} a_{i M+m}$ where $i$ is a multi-symbol block index and $1 \leq m \leq$ $M$.

(iii) Multi-Symbol Transmitted Reference (MSTR) with $b_{i(M+1)}=1$ and $b_{i(M+1)+m}=a_{i M+m}$ where $i$ is a multisymbol block index and $1 \leq m \leq M$.

In this paper, our focus is on the MSTR encoder in this paper. All these encoders employ the first modulated symbol as the reference signal in each block and the TR scheme [12] can be viewed as a special case of MSTR scheme where $M=1$. For MSDE case, the current transmitted symbols are encoded differentially with the previous encoded symbols and the first symbol is used as an initial symbol, while in MSTR case, the current transmitted symbol is the same as the information symbol except the first one, which is used to generate the reference template.

\section{GLRT-Based Multi-Symbol Detection}

In the case of multi-symbol detection, each block contains $M+1$ symbols including one reference symbol and $M$ 
information symbols. To simplify the equations in multisymbol detection cases, we consider only the encoding and detecting scheme in one block of $M+1$ symbols. Hence, the received signal can be rewritten as

$$
\begin{aligned}
x(t) & =\sum_{m=0}^{M} r_{m}\left(t-m T_{s}\right) \\
& =\sum_{m=0}^{M} b_{m} \sum_{j=0}^{N_{f}-1} g\left(t-m T_{s}-j T_{f}-c_{j} T_{c}\right)+n(t),
\end{aligned}
$$

by assuming that the channel is quasi-static over the interval $\left[0,(M+1) T_{s}\right]$.

Now, our task is to determine the information symbols $\mathbf{a}=\left[a_{0}, a_{1}, \ldots, a_{M}\right]^{T}, a_{0}=1$ without knowing the channel template $g(t)$. The relationship between information symbols $a_{m}$ and transmitted symbols $b_{m}$ for MSDE is

$$
b_{m}=\prod_{i=0}^{m} a_{i}, \quad \text { for } m=0, \ldots, M,
$$

and for MSTR is

$$
b_{m}=a_{m}, \quad \text { for } m=0, \ldots, M .
$$

Here, we resort to the generalized likelihood ratio test (GLRT) approach to detect the information symbols. The log-likelihood metric is given as

$$
\mathcal{L}(x(t) \mid \tilde{\mathbf{a}}, \tilde{g}(t))=-\int_{0}^{(M+1) T_{s}}(x(t)-\tilde{x}(t))^{2} d t
$$

where $\tilde{x}(t)$ is the candidate waveform constructed by $\tilde{\mathbf{a}}, \tilde{g}(t)$

$$
\begin{gathered}
\tilde{x}(t)=\sum_{m=0}^{M}\left(\mathbf{H}_{(m)} \otimes \widetilde{\mathbf{a}}\right) \sum_{j=0}^{N_{f}-1} \tilde{g}\left(t-m T_{s}-j T_{f}-c_{j} T_{c}\right), \\
\tilde{b}_{m}=\mathbf{H}_{(m)} \otimes \widetilde{\mathbf{a}},
\end{gathered}
$$

where $\mathbf{H}_{(m)}$ is the $(m+1)$ st row of an $(M+1) \times(M+1)$ matrix which comes from the encoding schemes (MSDE or MSTR) described in Section 2. All entries of $\mathbf{H}_{(m)}$ are 0 or 1 and $\left[h_{0}, h_{1}, h_{2}, \ldots, h_{M}\right] \otimes\left[a_{0}, a_{1}, a_{2}, \ldots, a_{M}\right]^{T}$ is defined as $\prod_{i=0, h_{i} \neq 0}^{M} a_{i}$. The $\mathbf{H}$ matrices for the MSDE and MSTR are

$$
\begin{aligned}
\mathbf{H}_{\mathrm{MSDE}} & =\left[\begin{array}{ccccccc}
1 & 0 & 0 & 0 & \cdots & 0 & 0 \\
1 & 1 & 0 & 0 & \cdots & 0 & 0 \\
1 & 1 & 1 & 0 & \cdots & 0 & 0 \\
1 & 1 & 1 & 1 & \cdots & 0 & 0 \\
\vdots & \vdots & \vdots & \vdots & \ddots & \vdots & \vdots \\
1 & 1 & 1 & 1 & \cdots & 1 & 0 \\
1 & 1 & 1 & 1 & \cdots & 1 & 1
\end{array}\right], \\
\mathbf{H}_{\mathrm{MSTR}} & =\left[\begin{array}{ccccccc}
1 & 0 & 0 & 0 & \cdots & 0 & 0 \\
1 & 1 & 0 & 0 & \cdots & 0 & 0 \\
1 & 0 & 1 & 0 & \cdots & 0 & 0 \\
1 & 0 & 0 & 1 & \cdots & 0 & 0 \\
\vdots & \vdots & \vdots & \vdots & \ddots & \vdots & \vdots \\
1 & 0 & 0 & 0 & \cdots & 1 & 0 \\
1 & 0 & 0 & 0 & \cdots & 0 & 1
\end{array}\right] .
\end{aligned}
$$

The decision rule of GLRT algorithm is of the form

$$
\widehat{\mathbf{a}}=\arg \max _{\widetilde{\mathbf{a}}, \tilde{g}(t)}\{\mathcal{L}(x(t) \mid \widetilde{\mathbf{a}}, \tilde{g}(t))\} .
$$

In (10), although $g(t)$ is unknown, it is treated as a nuisance parameter. The optimum reference template given a symbol candidate $\tilde{\mathbf{a}}$ can be obtained using the variational technique (see [17])

$$
\begin{aligned}
\hat{g}(t) & =\arg \max _{\tilde{g}(t)}\{\mathcal{L}(x(t) \mid \tilde{\mathbf{a}}, \tilde{g}(t))\} \\
& =\frac{1}{M+1} \sum_{m=0}^{M}\left(\mathbf{H}_{(m)} \otimes \tilde{\mathbf{a}}\right) y_{m}(t),
\end{aligned}
$$

where $y_{m}(t)$ is the averaged waveform for the $m$ th received symbol signal over $N_{f}$ frames

$$
\begin{aligned}
y_{m}(t) & =\frac{1}{N_{f}} \sum_{j=0}^{N_{f}-1} x\left(t+m T_{s}+j T_{f}+c_{j} T_{c}\right), \quad 0 \leq t \leq T_{f} \\
& =\frac{1}{N_{f}} \sum_{j=0}^{N_{f}-1} r_{m}\left(t+j T_{f}+c_{j} T_{c}\right), \quad 0 \leq t \leq T_{f} .
\end{aligned}
$$

Incorporating the log-likelihood formula in (10) and (11), finally we have

$$
\widehat{\mathbf{a}}=\arg \max _{\widetilde{\mathbf{a}}} \sum_{m=0}^{M-1} \sum_{l=m+1}^{M}\left(\mathbf{H}_{(m)} \otimes \widetilde{\mathbf{a}}\right)\left(\mathbf{H}_{(l)} \otimes \widetilde{\mathbf{a}}\right) Z_{m, l},
$$

where

$$
Z_{i, j}=\int_{0}^{T_{r}} y_{i}(t) y_{j}(t) d t
$$

where $T_{r}$ is the integration interval of the correlator, and $T_{r} \leq T_{m}+T_{p}$.

Some remarks are now of interest.

(i) For the single user or multiple-orthogonal users case with $M=1,(13)$ reduces to

$$
\hat{a}_{1}=\arg \max _{\widetilde{a}_{1}} \tilde{a}_{1} Z_{0,1},
$$

which is equivalent to averaged transmitted reference (ATR) detection for single symbol detection [12]

$$
\hat{a}_{1}=\operatorname{sgn}(z)=\operatorname{sgn}\left(Z_{0,1}\right),
$$

where $z=Z_{0,1}$ is the decision variable for ATR.

Simple mathematical manipulations yield the following expressions for the mean and variance of the decision variable $z$ as

$$
\begin{gathered}
\mu=\mathrm{E}\{z\}=a_{i} E_{f} \\
\sigma^{2}=\operatorname{Var}\{z\}=\frac{E_{f} N_{0}}{N_{f}}+\frac{N_{0}^{2} T_{r} W}{2 N_{f}^{2}}
\end{gathered}
$$


where $W$ is the one-sided noise bandwidth of the receiver, $\mathrm{E}\{\cdot\}$ is the statistical expectation, and $\operatorname{Var}\{\cdot\}$ is the variance of the random variable. The BER of the detector in this case is [12]

$$
P_{\text {ATR }}=Q\left(\left[\frac{N_{0}}{N_{f} E_{f}}+\frac{N_{0}^{2} T_{r} W}{2 N_{f}^{2} E_{f}^{2}}\right]^{-1 / 2}\right),
$$

where $Q(x)$ is the $Q$-function $Q(x)=(2 \pi)^{-1 / 2} \int_{x}^{\infty} \exp \left(-t^{2} /\right.$ 2) $d t$.

(ii) Unlike the ideal Rake receiver, which correlates the receive signal with noiseless template, the TR scheme uses the noisy reference signal as a template in one symbol case and the best estimated reference signal using (11) in the multi-symbol case. However, the TR system does not explicitly estimate the channel parameters and only requires the correlation coefficients $Z_{m, l}, Z_{m, l}=Z_{l, m}, 0 \leq m \leq M$, $0 \leq l \leq M$ evaluated in (14).

(iii) As seen in (11), the variance of the reference signal $\hat{g}(t)$ decays as $M$ increases when $\tilde{\mathbf{a}}=\mathbf{a}$. In turn, the accuracy of the multi-symbol detection is improved and converges to the performance of ideal Rake receiver as $M \rightarrow \infty$.

(iv) The global optimal value of $\hat{\mathbf{a}}$ can be obtained by using exhaustive search [16] or sphere decoding [17]. However, the computational cost of the exhaustive search method grows exponentially with the number of symbols $M$. Sphere decoding method searches all the lattice points inside a given radius and reduces the complexity of the exhaustive search method on average. However, the expected complexity of SDA is still exponential for fixed SNR and increases significantly when SNR is low [18].

\section{A Fast MSTR Detection Method}

In this section, we develop an iterative MSTR detection algorithm by avoiding the high computational complexity of GLRT-based detectors (e.g., exhaustive search [16] and SDA [17]). Similar to the TR detection scheme, the proposed method first generates a reference template by using the initial symbol only, and then estimates the information symbols by correlating the reference template with the symbol waveforms. Furthermore, with the help of the information from multiple transmitted symbols, our method manages to suppress the reference template noise. However, our method also generates additional noise-cross-signal and noise-crossnoise terms which do not appear in the case of an ideal Rake receiver with perfect channel knowledge.

For the initialization, since the only known symbol is $b_{0}=1$, the best template at this stage is

$$
\tilde{g}^{(1)}(t)=b_{0} y_{0}(t)=y_{0}(t)
$$

where $y_{0}(t)$ can be found in (12).
The decision variables for the $M$ information symbols are

$$
\begin{aligned}
z_{m}^{(1)} & =\int_{0}^{T_{r}} \tilde{g}^{(1)}(t) y_{m}(t) d t \\
& =\int_{0}^{T_{r}} y_{0}(t) y_{m}(t) d t \\
& =Z_{0, m}, \quad \text { for } m=1, \ldots, M .
\end{aligned}
$$

The estimated information symbols in this iteration are

$$
\tilde{b}_{m}^{(1)}=\operatorname{sgn}\left(Z_{0, m}\right), \quad \text { for } m=1, \ldots, M .
$$

This means that at the first step the estimated symbols are obtained by correlating the waveform corresponding to $b_{0}$ with the $m$ th symbol waveform. Hence, the BER performance is the same as that of the ATR in (19).

For the $n$th iteration, the method firstly constructs a new reference template by weighting the product of each symbol's waveform $y_{i}(t)$ and its corresponding detected symbol $\tilde{b}_{i}^{(n-1)}$ obtained from the previous iteration

$$
\tilde{g}^{(n)}(t)=w_{0}^{(n-1)} y_{0}(t)+\sum_{i=1}^{M} w_{i}^{(n-1)} \tilde{b}_{i}^{(n-1)} y_{i}(t) .
$$

Then, the decision variable for the $m$ th symbol is evaluated in the same way as the one in (21)

$$
\begin{aligned}
z_{m}^{(n)} & =\int_{0}^{T_{r}} \tilde{g}_{m}^{(n)}(t) y_{m}(t) d t \\
& =w_{0}^{(n-1)} Z_{0, m}+\sum_{i=1, i \neq m}^{M} w_{i}^{(n-1)} \tilde{b}_{i}^{(n-1)} Z_{i, m},
\end{aligned}
$$

where $\widetilde{g}_{m}^{(n)}(t)$ is the reference template for the $m$ th symbol by removing the $m$ th waveform $y_{m}(t)$ from $\tilde{g}^{(n)}(t)$

$$
\tilde{g}_{m}^{(n)}(t)=w_{0}^{(n-1)} y_{0}(t)+\sum_{i=1, i \neq m}^{M} w_{i}^{(n-1)} \widetilde{b}_{i}^{(n-1)} y_{i}(t) .
$$

At last, the iteration outputs the estimated symbols as

$$
\tilde{b}_{m}^{(n)}=\operatorname{sgn}\left(z_{m}^{(n)}\right)
$$

4.1. Weight Selections. A key factor that affects the method's performance and convergence is how to update the weights in each iteration. The ultimate goal of selecting the weights is to reduce BER while maintaining low computational complexity and requiring little extra knowledge (such as channel information). Here, we propose two types of rule for the choice of the weights in each iteration.

(i) Hard Decision for MSTR (HD-MSTR). The rule constructs the reference template as

$$
\tilde{g}^{(n)}(t)=y_{0}(t)+\sum_{i=1}^{M} \tilde{b}_{i}^{(n-1)} y_{i}(t),
$$


which indicates that $\mathbf{w}^{(n-1)}=[1,1, \ldots, 1]$ in (23). Also note that, the template is a scaled version of the GLRT template estimate given the detected symbols $\widetilde{b}^{(n-1)}$ as shown in (11).

An interesting observation on the reference template of HD-MSTR in (27) is that the variance of the reference template is constant given the detected symbols $\widetilde{b}_{m}^{(n-1)}$

$$
\begin{aligned}
\operatorname{Var}\left\{\tilde{g}^{(n)}(t)\right\} & =\operatorname{Var}\left\{y_{0}(t)\right\}+\sum_{i=1}^{M} \operatorname{Var}\left\{\tilde{b}_{i}^{(n-1)} y_{i}(t)\right\} \\
& =(M+1) \operatorname{Var}\left\{y_{0}(t)\right\}
\end{aligned}
$$

The conditional mean of the template is

$$
\begin{aligned}
\mathrm{E}\left\{\tilde{g}^{(n)}(t) \mid \mathbf{b}\right\} & =g(t)+\sum_{m=1}^{M}\left\{\hat{b}_{m}^{(n-1)} b_{m}\right\} g(t) \\
& =\left(1+2 N_{c}^{(n-1)}-M\right) g(t),
\end{aligned}
$$

where $N_{c}^{(n-1)}$ is the number of correct symbols for the $(n-$ 1)st iteration. Hence, the mean and standard deviation ratio is

$$
\frac{\mathrm{E}\left\{\tilde{g}^{(n)}(t) \mid \mathbf{b}\right\}}{\operatorname{Std}\left\{\tilde{g}^{(n)}(t)\right\}}=\frac{\left(1+2 N_{c}^{(n-1)}-M\right) g(t)}{\sqrt{(M+1)} \operatorname{Std}\left\{y_{0}(t)\right\}},
$$

where $\operatorname{Std}\{\cdot\}$ is the standard deviation of the random variable. In general, the larger the mean-standard deviation ratio, the better the BER performance. Thus, in the case of HD-MSTR, if more correct symbols are detected for the current iteration, during the next iteration, the reference template is improved and then the method potentially results in better BER performance. The iterative procedure runs back and forth until no symbol is changed or the maximum number of iterations is reached.

(ii) Soft Decision for MSTR (SD-MSTR). An intuitive idea of the SD-MSTR detector is that the decision variable $z_{m}^{(n)}$ obtained in each iteration reflects the reliability of the detected symbol $\tilde{b}_{m}^{(n)}$. The larger the value of $z_{m}^{(n)}$, the more we can trust the accuracy of the detected symbol $\tilde{b}_{m}^{(n)}$. Hence, the corresponding symbol deserves higher weight in the representation of the reference template for next iteration.

By facilitating the additional information from decision variables $z_{m}^{(n)}$, the SD-MSTR determines the weight values as

$$
\begin{aligned}
w_{m}^{(n)} & =P\left(b_{m}=\tilde{b}_{m}^{(n)} \mid z_{m}^{(n)}\right)-P\left(b_{m} \neq \tilde{b}_{m}^{(n)} \mid z_{m}^{(n)}\right) \\
& =2 P\left(b_{m}=\tilde{b}_{m}^{(n)} \mid z_{m}^{(n)}\right)-1, \quad \text { for } m=0, \ldots, M,
\end{aligned}
$$

where the two terms in (31) are the posterior probabilities of correct and erroneous detection of the symbol $\tilde{b}_{m}^{(n)}$, conditioned on the decision variable $z_{m}^{(n)}$. If these probabilities are the same, that means it does not matter which decision we make. This represents the most unreliable case and the weight should be set to zero. The larger the probability of correct detection, the higher weight we should put on this decision. Note that the weight $w_{0}^{(n)}$ of the known reference symbol $b_{0}$ is set to $1, w_{m}^{(n)}$ should be $0 \leq w_{m}^{(n)} \leq 1$ and $w_{m}^{(n)} \tilde{b}_{m}^{(n)}$ ranges from $[-1,+1]$ indicating how much the averaged signal $y_{m}(t)$ contributes to the final template depending on accuracy of the estimated symbol $\tilde{b}_{m}^{(n)}$.

By applying Bayes' rule, (31) becomes

$$
w_{m}^{(n)}=\frac{P\left(z_{m}^{(n)} \mid b_{m}=\tilde{b}_{m}^{(n)}\right)-P\left(z_{m}^{(n)} \mid b_{m} \neq \tilde{b}_{m}^{(n)}\right)}{P\left(z_{m}^{(n)} \mid b_{m}=\tilde{b}_{m}^{(n)}\right)+P\left(z_{m}^{(n)} \mid b_{m} \neq \tilde{b}_{m}^{(n)}\right)},
$$

$$
\text { for } m=0, \ldots, M \text {, }
$$

where the probabilities rely on the distribution of $z_{m}^{(n)}$ which is approximately Gaussian distributed with mean $b_{m} \mu_{m}^{(n)}\left(\mu_{m}^{(n)}>0\right)$, and variance $\left(\sigma_{m}^{(n)}\right)^{2}$ given $b_{m}[13]$

$$
\begin{aligned}
P\left(z_{m}^{(n)} \mid b_{m}=+1\right) & =\frac{1}{\sqrt{2 \pi} \sigma_{m}^{(n)}} \exp \left(-\frac{\left(z_{m}^{(n)}-\mu_{m}^{(n)}\right)^{2}}{2\left(\sigma_{m}^{(n)}\right)^{2}}\right) \\
& =\frac{1}{\sigma_{m}^{(n)}} \phi\left(\frac{z_{m}^{(n)}-\mu_{m}^{(n)}}{\sigma_{m}^{(n)}}\right), \\
P\left(z_{m}^{(n)} \mid b_{m}=-1\right) & =\frac{1}{\sqrt{2 \pi} \sigma_{m}^{(n)}} \exp \left(-\frac{\left(z_{m}^{(n)}+\mu_{m}^{(n)}\right)^{2}}{2\left(\sigma_{m}^{(n)}\right)^{2}}\right) \\
& =\frac{1}{\sigma_{m}^{(n)}} \phi\left(\frac{z_{m}^{(n)}+\mu_{m}^{(n)}}{\sigma_{m}^{(n)}}\right)
\end{aligned}
$$

where $\phi(x)=(1 / \sqrt{2 \pi}) e^{-(1 / 2) x^{2}}$ is the probability density function (pdf) of the standard normal distribution.

A practical issue in SD-MSTR is how to evaluate the statistics of $z_{m}^{(n)}$ in each iteration since we do not have an explicit formula. An approximate solution of the problem is to utilize the known ATR statistics to evaluate the probabilities for each iteration

$$
\begin{aligned}
& P\left(z_{m}^{(n)} \mid b_{m}=+1\right) \approx \frac{1}{\sigma} \phi\left(\frac{z_{m}^{(n)}-\mu}{\sigma}\right), \\
& P\left(z_{m}^{(n)} \mid b_{m}=-1\right) \approx \frac{1}{\sigma} \phi\left(\frac{z_{m}^{(n)}+\mu}{\sigma}\right),
\end{aligned}
$$

where $\mu$ and $\sigma$ can be found in (17) and (18) which require the frame energy $E_{f}$ and the noise power $N_{0}$ to evaluate $\mu$ and $\sigma$, but they are easy to estimate and store at the receiver side.

Now, we can summarize our method in the following steps for one block symbol detection.

Input: Correlation matrix $Z_{i, j}$ defined in (14), where $0 \leq$ $i \leq M, 0 \leq j \leq M$, the maximum number of iterations $N$, channel statistics $\mu$ and $\sigma$ for the SD-MSTR case. 
Step 1. Initialize $\mathbf{w}^{(0)}=[1,1, \ldots, 1], \tilde{\mathbf{b}}^{(0)}=[1,0, \ldots, 0]$, $n=0$.

Step 2. $n=n+1$.

Step 3. Obtain the decision variables by (24).

Step 4. Obtain the detected symbols by (26).

Step 5. Set $w^{(n)}=w^{(0)}$ for the HD-MSTR case or update the weights for $\mathbf{w}^{(n)}$ based on (31), (35), (36) in the SD-MSTR case.

Step 6. If $n<N$ and $\tilde{\mathbf{b}}^{(n)} \neq \tilde{\mathbf{b}}^{(n-1)}$ goto Step 2, otherwise output $\tilde{\mathbf{b}}^{(n)}$ and exit.

4.2. Convergence and Discussions. (i) The convergence rate also affects the practical value of the method (e.g., a system with a tight constraint on decoding delay) and the number of iterations affects the performance. These will be verified by the numerical simulation that the proposed method converges to the stable performance curve within a few iterations (usually $\leq 5$ iterations).

(ii) Comparing with MSDD, we choose MSTR as the encoding scheme which allows the algorithm to detect symbol $b_{m}=a_{m}$ directly without any further processing.

(iii) Instead of evaluating each iteration's reference template $\tilde{g}^{(n)}(t)$ explicitly, the method computes the decision variables by linear combination of the correlation coefficients $Z_{i, j}$ which can be computed in the first iteration and reused later.

(iv) The HD-MSTR only requires the coefficients $Z_{i, j}$ which is the same as the GLRT approach meanwhile the SD-MSTR requires some additional channel statistical information to update the weights for each iteration.

(v) For each iteration, Step 3 requires $2 M(M-1)$ multiplications and $M^{2}$ additions to attain the decision variables for all $M$ symbols. In Step $4, M$ sign operations are performed to obtain detected symbols. No arithmetic is required for HD-MSTR in Step 5, while the SD-MSTR performs $2 M$ times Gaussian pdf evaluation and needs $3 M$ additions and $M$ divisions to normalize weights. We can treat the computational costs of sign operation and Gaussian pdf evaluation as being constant, and then the computational complexity of the both detectors for each iteration is $\mathcal{O}\left(M^{2}\right)$ where $M$ is the block size. Note that the complexity of the proposed method is independent of the channel realizations whilst the computational complexity of SDA relies on the specific realization of channels and SNR.

\section{Numerical Results}

This section compares the BER performance of the proposed methods (HD-MSTR and SD-MSTR) and the MSTR based on exhaustive search (ES-MSTR) as benchmark. Two kinds of channel schemes are evaluated: one is a constant channel with fixed CIR parameters, and the other is a random channel based on Saleh and Valenzuela (SV) channel model.

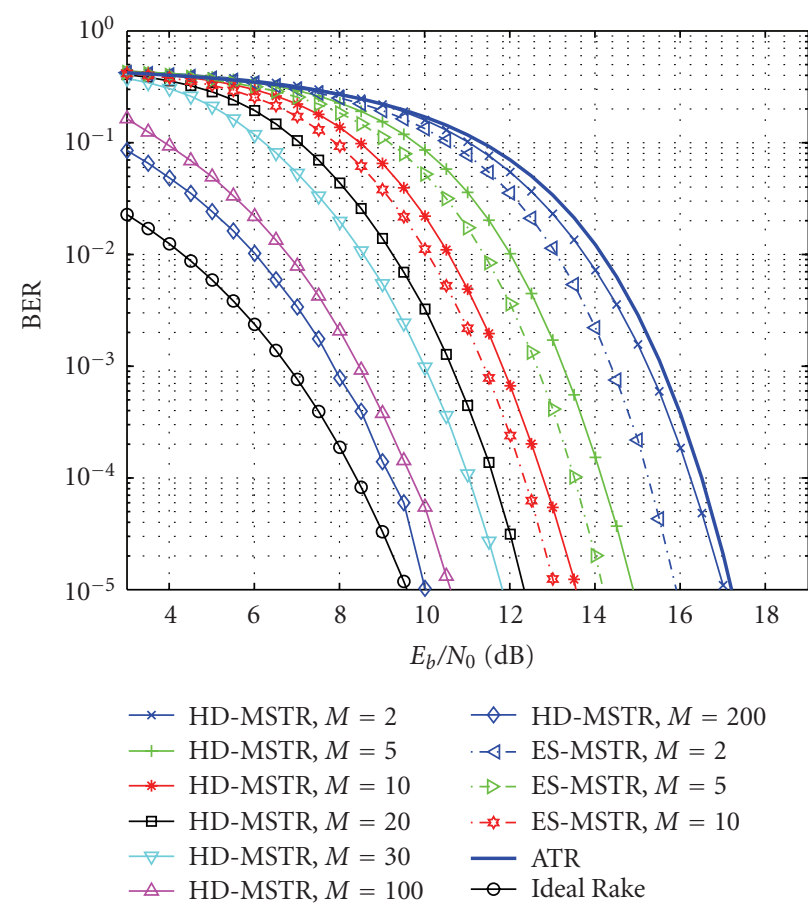

Figure 1: BER of HD-MSTR for different $M, K=200, N_{f}=20$, $N=10$.

5.1. Constant Channel. At the transmitter side, the pulse $p(t)$ is the second derivative of a Gaussian function with normalized unit energy and pulse width $T_{p}=1.0 \mathrm{~ns}$. The number of frames per symbols is $N_{f}=20$. For the UWB channel model, we employ the resolvable multipath assumption such that $\tau_{k}=k T_{p}$ as studied in $[12,13,19]$ and then $W T_{r}$ in (18) can be approximated with the number of paths $K$. In this simulation, $K$ is 200 and the energy of impulse channel response (CIR) $\int_{0}^{T_{f}}|h(t)|^{2} d t=1$ which means $E_{f}=E_{p}$ in this scheme. As we have shown in Section 3, if the number of symbols in one block $M$ is equal to 1 or the maximum number of iterations $N$ is equal to 1 , then the system outputs the same performance as ATR scheme in [12]. Note that there is a $3 \mathrm{~dB}$ gap between the ATR curve in the following figures and the one in [12]. This is because the definition of frame energy in [12] is twice as the one of ours. In this subsection, we only consider single user case with $c_{j}=0$, for all $j$. Multiuser case will be shown in next subsection.

5.1.1. BER with Different Block Size. Figures 1 and 2 illustrate the BER results for $M=2,5,10,20,30,100,200$ for HDMSTR and SD-MSTR, respectively. For HD-MSTR, the proposed method can obtain about $2 \mathrm{~dB}$ gain relative to ATR in the case of $M=5$ and about $3 \mathrm{~dB}$ gain if $M=10$. With the increase of the number of symbols in one block, the performance of the proposed method grows monotonically but the improvement decelerates ( $5 \mathrm{~dB}$ gain for $M=20$ and $5.3 \mathrm{~dB}$ gain for $M=30$ ). In the same figure, we also depict the performance of the GLRT algorithm with exhaustive search (called ES-MSTR) as benchmarks. We also perform 


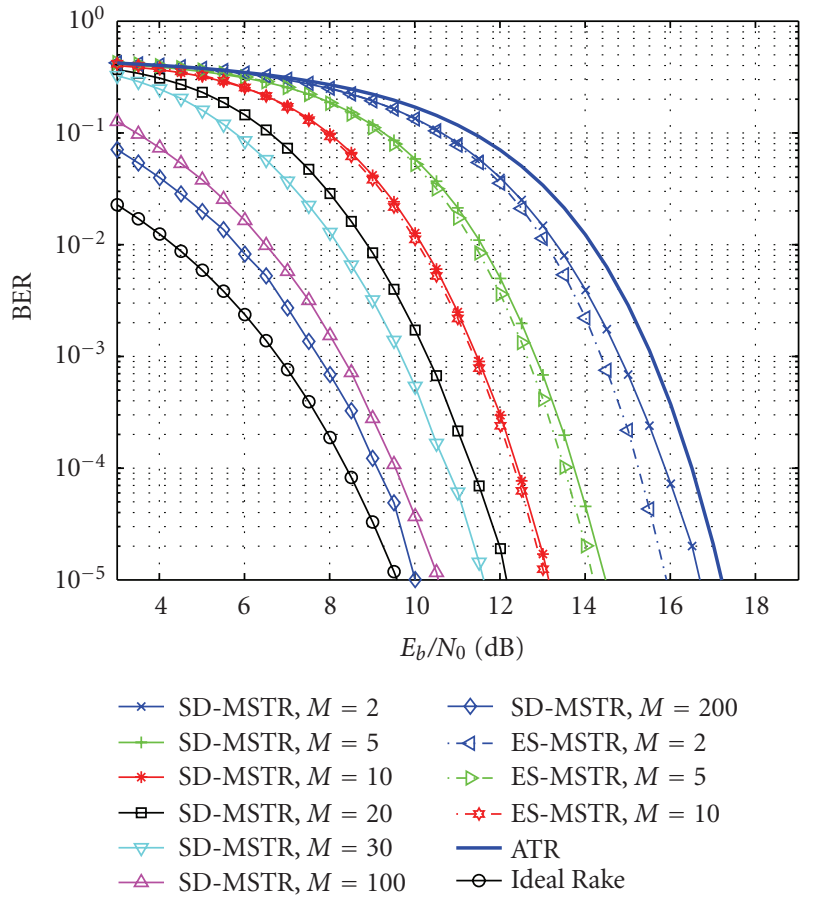

Figure 2: BER of SD-MSTR for different $M, K=200, N_{f}=20$, $N=10$.

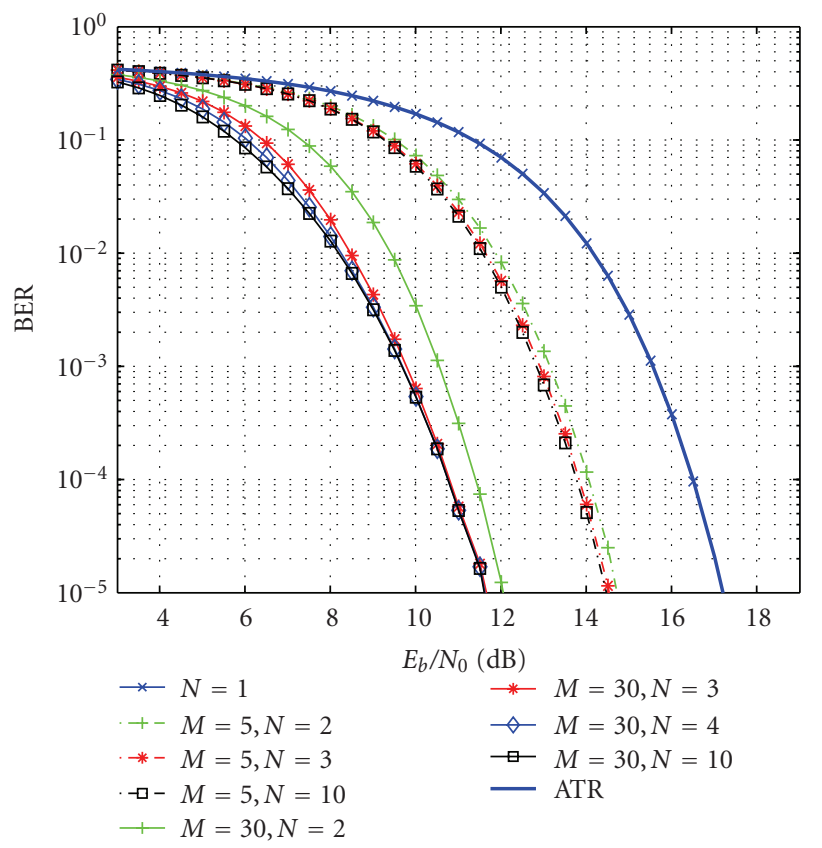

Figure 3: BER of HD-MSTR for different iterations $M=5,30$, $K=200, N_{f}=20$.

some simulations with very large $M(M=100,200)$ which is intractable for classical methods. The system provides similar performance to that of the ideal Rake receiver, especially in high SNR range, where the difference is less than $1 \mathrm{~dB}$.

Comparing the performance of HD-MSTR and SDMSTR detectors in Figures 1 and 2, respectively, the difference is obvious when $M$ is small. The SD-MSTR outperforms

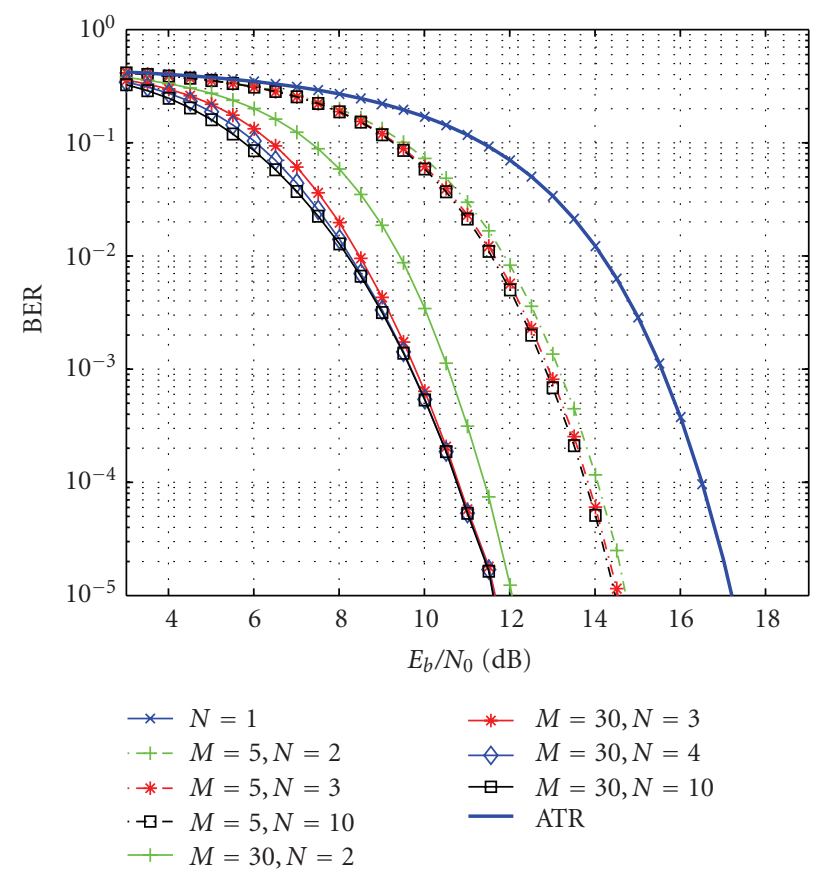

FIGURE 4: BER of SD-MSTR for different iterations $M=5,30, K=$ 200, $N_{f}=20$.

the HD-MSTR, with about $0.5 \mathrm{~dB}$ of SNR gain when $M=$ 5,10 and around $0.2 \mathrm{~dB}$ gain when $M=20,30$. The difference becomes trivial when $M$ is 100 or larger. This indicates that the SD-MSTR method can offer additional advantages for low complexity UWB systems with small $M$ and but its advantage decreases with increasing $M$. Bearing in mind that the SD-MSTR requires some statistical channel information ( $\mu, \sigma$ in (17) and (18)) and the Gaussian pdf calculation of the system, it is more likely that the simpler HD-MSTR algorithm would be implemented if $M$ is large.

Compared with HD-MSTR and SD-MSTR, the ESMSTR has an advantage when $M$ is small (if $M=2$, about $1.1 \mathrm{~dB}$ gain for HD-MSTR and $0.6 \mathrm{~dB}$ for SD-MSTR) and the performance gap becomes smaller when $M$ is larger. When $M=10$, the gap reduces to around $0.5 \mathrm{~dB}$ for HD-MSTR case and about $0.1 \mathrm{~dB}$ for the SD-MSTR case. This shows that with the increasing value of $M$ the difference between the optimal ES-MSTR method and our proposed methods decreases rapidly and that the gap can be ignored for a sufficient large $M$. Furthermore, the ES-MSTR incurs much higher computational cost than our MSTR method.

5.1.2. BER with Different Iterations. To answer the convergence question in Section 4.2, Figures 3, 4, 5, and 6 depict the BER values recorded in each iteration for $M=5,30$. When there is only one iteration, the system reduces to classic ATR system and the BER result overlaps with that given by (19) (see Figures 3 and 4). The BER is improved significantly in the second iteration and just after about 4 iterations, the algorithm reaches a stable BER performance curve with a small improvement in the 5th iteration at low SNRs. These show that our methods converge fast and it is practical for 


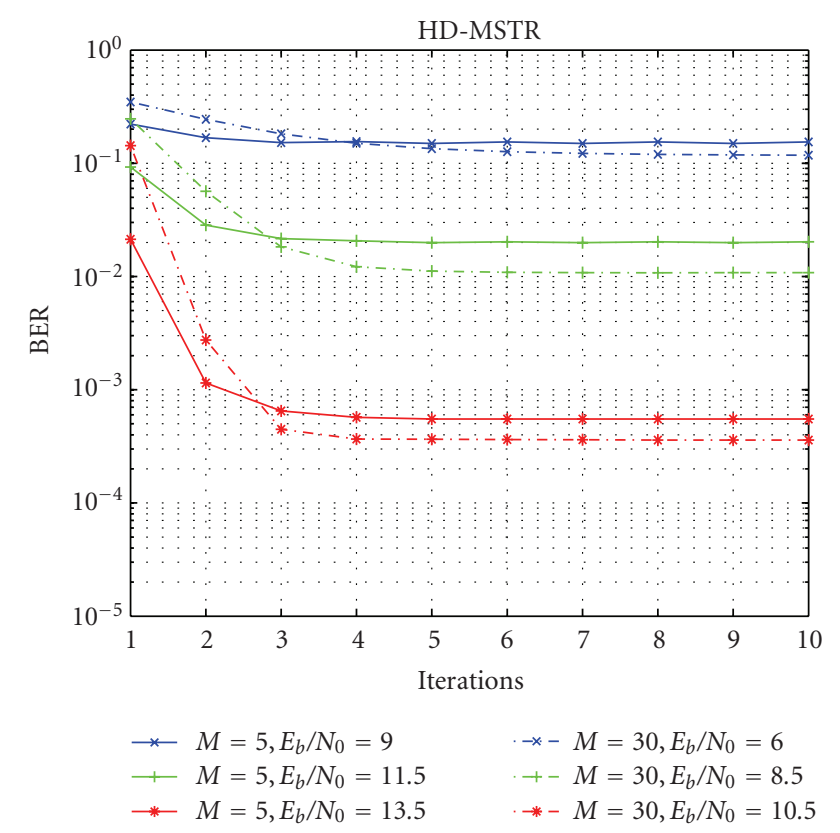

FIgURe 5: Convergence rate of HD-MSTR with $M=5,30$ in different SNR level.

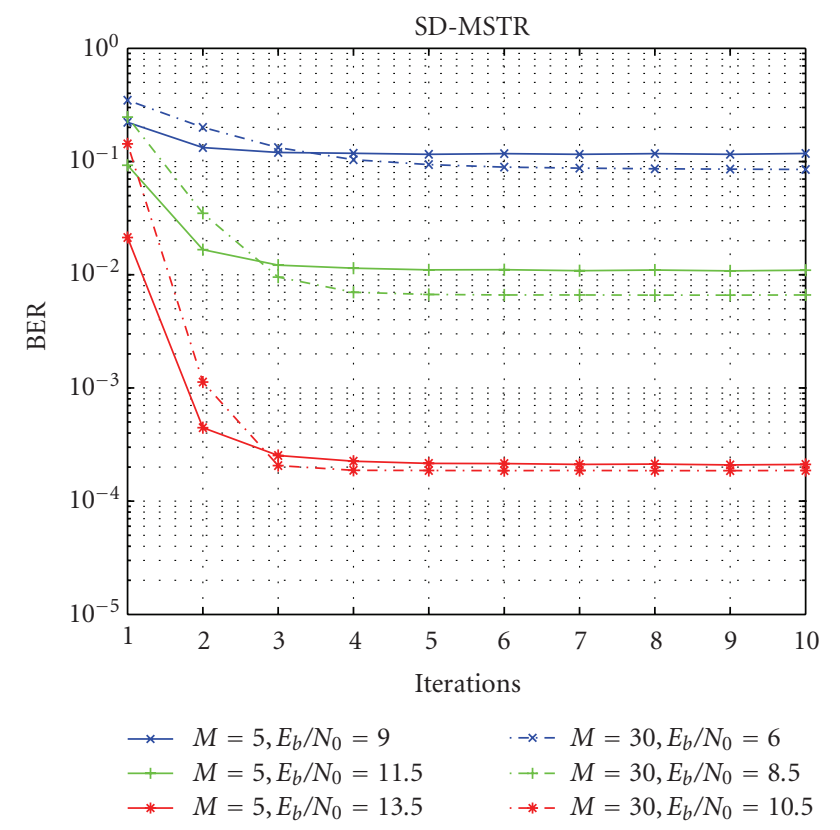

FIgURE 6: Convergence rate of SD-MSTR with $M=5,30$ in different SNR level.

UWB systems. It is also noticed that the HD-MSTR and SDMSTR show the similar convergence rates in the figures.

5.2. SV Channel Model. The more realistic UWB channel is random which significantly affects the BER performance compared with constant channels. The SV channel model which is generally statistically verified to well describe the realistic UWB channel with dense multipath is adopted in

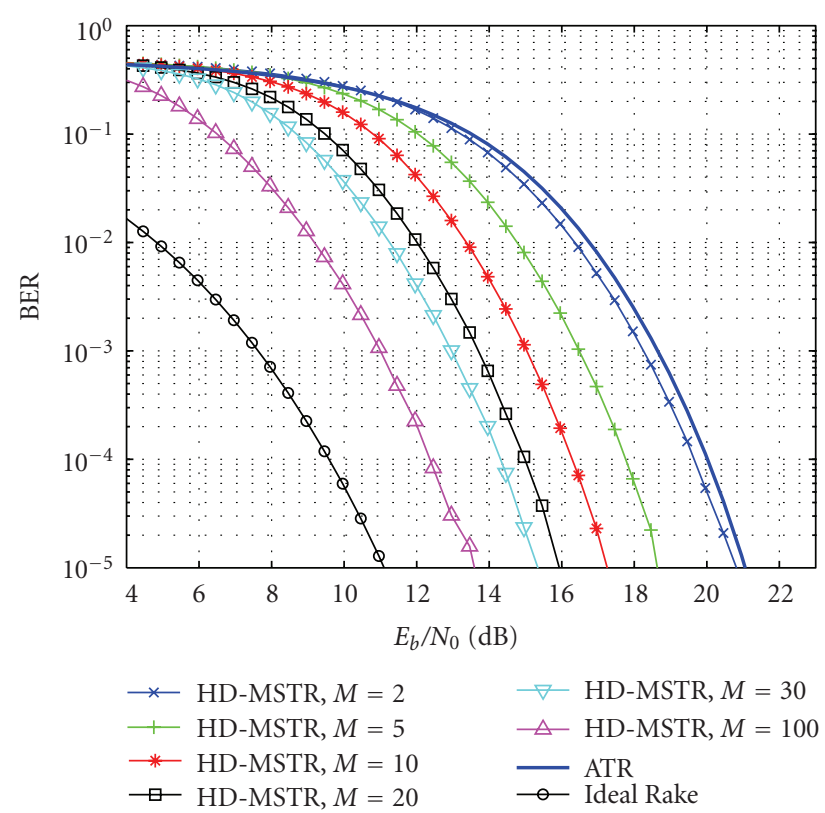

FIGURE 7: Average BER of HD-MSTR for different $M$ over random channels, $N=10$.

this section. The model formulates the channel impulse response (CIR) as [20]

$$
h(t)=\sum_{l=0}^{\infty} \sum_{k=0}^{\infty} \beta_{k l} p_{k l} \delta\left(t-T_{l}-\tau_{k l}\right),
$$

where $\beta_{k l} p_{k l}$ models the double-sided Rayleigh distributed amplitudes with exponentially decaying profile.

In our experiments, the SV channel model parameters are: $\Gamma=30 \mathrm{~ns}, \gamma=5 \mathrm{~ns}, \Lambda=0.5 \mathrm{~ns}^{-1}, \lambda=2 \mathrm{~ns}^{-1}$ (see [20, (23), (24), (26)] ). The pulse $p(t)$ is the monocycle which is the same as the one in Section 5.1. The energy per bit $E_{b}$ is defined as

$$
E_{b}=N_{f} \overline{E_{f}}
$$

where $\overline{E_{f}}$ is the average received energy per frame and the frame repetition factor is $N_{f}=25$ (to compare with [17]) while the integral interval is $T_{r}=100 \mathrm{~ns}$ and the frame duration is $T_{f}=200$ ns to preclude the IFI.

5.2.1. Single User Scenario. Figures 7 and 8 plot the performance curves for both HD-MSTR and SD-MSTR in single user scenario, respectively. For random channels, SD-MSTR shows about $0.5 \mathrm{~dB}$ gain over the $\mathrm{HD}-\mathrm{MSTR}$ for $M=$ $2,5,10,20,30$ and retains the advantage even for large $M$ $(M=100)$. For HD-MSTR case, the algorithm achieves $2 \mathrm{~dB}$ gain if $M=5$ and about $6 \mathrm{~dB}$ gain if $M=30$ with respect to ATR. This means that with a few iterations, the algorithms efficiently exploit the multi-symbol benefits and yield a near optimal result. Furthermore, by avoiding searching the solution space which is computational complex, our iterative methods are easy to compute by adding up some correlation terms with different weights in (24). 


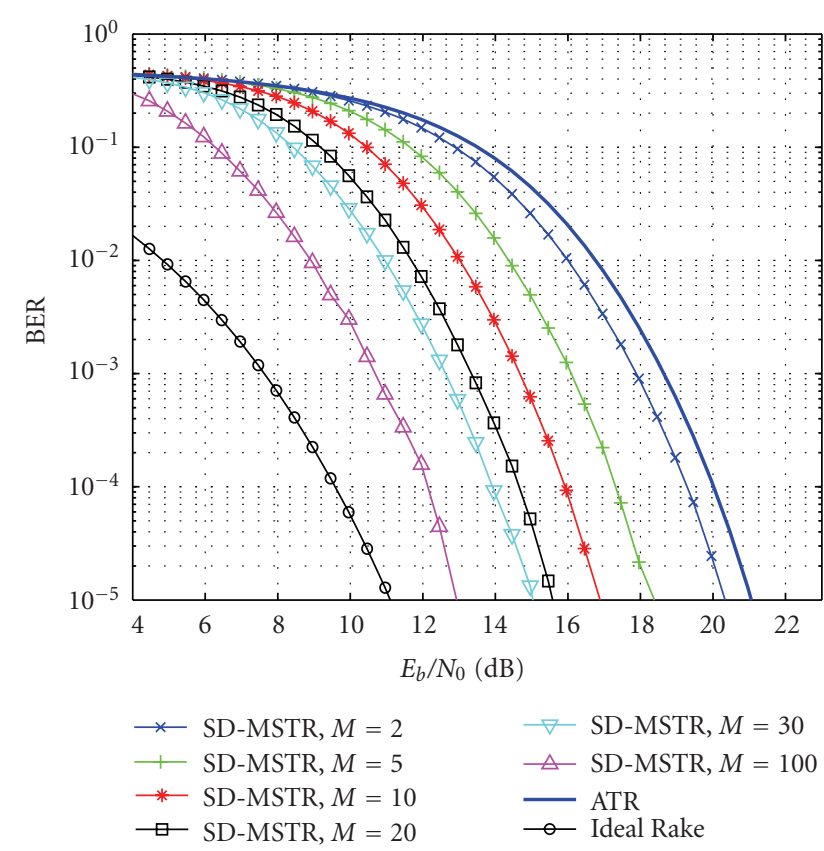

FIGURe 8: Average BER of SD-MSTR for different $M$ over random channels, $N=10$.

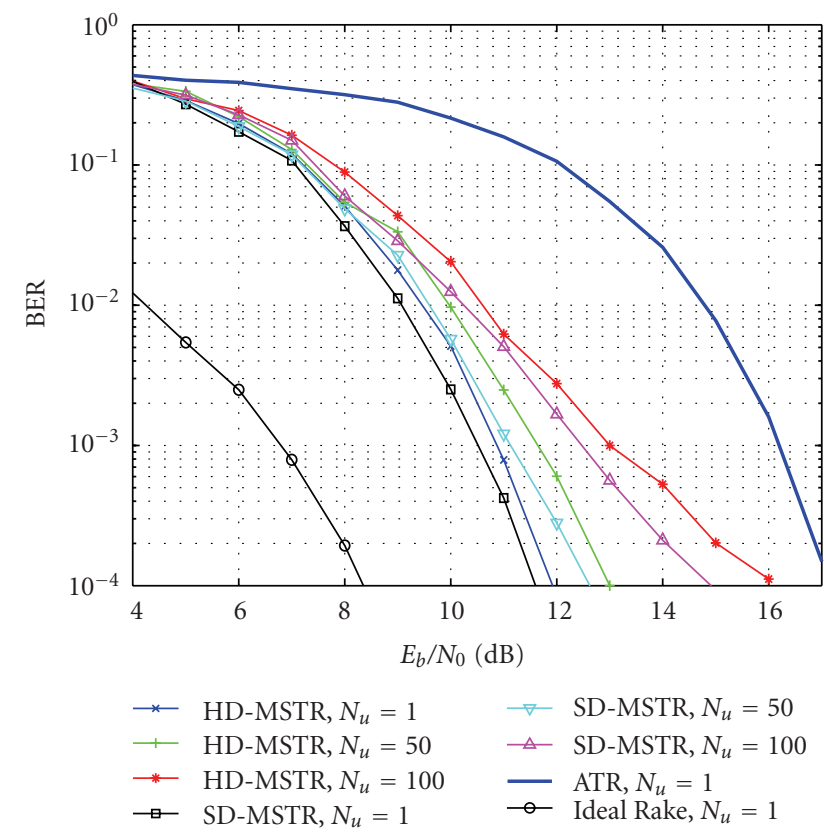

Figure 9: BER of HD-MSTR and SD-MSTR for $M=30, N=10$, and different $N_{u}$ over random channels.

Furthermore, when $M=100$ and $\mathrm{BER}=10^{-5}$, there is about $2 \mathrm{~dB}$ gap between the ideal Rake receiver and our algorithm. As we expected, the performance over random channels is worse than the one with constant channels.

5.2.2. Multiuser Scenario. In this subsection, we consider the performance of our algorithms in the presence of multiple access interference (MAI). In the case of MAI, the chip interval is $T_{c}=1.0 \mathrm{~ns}$ and the $\mathrm{TH}$ codes $c_{j}$ are randomly generated in the range $\left[0, N_{c}-1\right]$ where $N_{c}=91$. Unlike the single user scenario, we do not consider the attenuation of each individual channel and assume ideal power control among nodes such that the received energy from each interfering user is the same. Figure 9 displays the BER result in this MAI scenario. At BER $=10^{-4}$, the HD-MSTR experiences only around $1 \mathrm{~dB}$ performance degradation for $N_{u}=50$ comparing with corresponding single user scenario where $N_{u}$ is the number of users. In addition, there is less than $5 \mathrm{~dB}$ gap between the multiple users HD-MSTR and single user ideal Rake receiver. For the SD-MSTR case, the detector outperforms the HD-MSTR detector with more than $0.2 \mathrm{~dB}$ gain in both single and multiple users scenario. In summary, our proposed detectors demonstrate significant robustness in the present of the MAI effects.

\section{Conclusion}

In this paper, we propose fast detection methods for MSTR transmissions. The proposed MSTR detectors obtain the decision variables by summing up the correlation of different symbol waveforms, each properly weighted by the reliability of detected symbols and iteratively updating the weights and detected symbols. With different updating methods for the weights, two detectors are proposed: Hard Decision for MSTR (HD-MSTR) detector and Soft Decision for MSTR (SD-MSTR) detector, where HD-MSTR obtains the template based only on the previous detected symbols, while SDMSTR constructs the template with additional information from the decision variables. Enhanced BER performance relative to the ATR scheme and the fast convergence property of these detectors are shown by the simulation results. Due to its simplicity, low computational complexity and near optimal performance for $M \geq 10$, the method is promising for realistic UWB applications.

\section{Acknowledgments}

Part of this work is supported by the Georgia Tech Ultrawideband Center of Excellence (http://www.uwbtech .gatech.edu/). The authors would like to thank the anonymous reviewers and the guest editor for their helpful comments which improved the quality of this paper.

\section{References}

[1] M. Z. Win and R. A. Scholtz, "Impulse radio: how it works," IEEE Communications Letters, vol. 2, no. 2, pp. 36-38, 1998.

[2] M. Z. Win and R. A. Scholtz, "Ultra-wide bandwidth timehopping spread-spectrum impulse radio for wireless multipleaccess communications," IEEE Transactions on Communications, vol. 48, no. 4, pp. 679-691, 2000.

[3] L. Yang and G. B. Giannakis, "Ultra-wideband communications: an idea whose time has come," IEEE Signal Processing Magazine, vol. 21, no. 6, pp. 26-54, 2004.

[4] R. J.-M. Cramer, R. A. Scholtz, and M. Z. Win, "Evaluation of an ultra-wide-band propagation channel," IEEE Transactions on Antennas and Propagation, vol. 50, no. 5, pp. 561-570, 2002. 
[5] M. Z. Win and R. A. Scholtz, "Characterization of ultrawide bandwidth wireless indoor channels: a communicationtheoretic view," IEEE Journal on Selected Areas in Communications, vol. 20, no. 9, pp. 1613-1627, 2002.

[6] D. Cassioli, M. Z. Win, and A. F. Molisch, "The ultra-wide bandwidth indoor channel: from statistical model to simulations," IEEE Journal on Selected Areas in Communications, vol. 20, no. 6, pp. 1247-1257, 2002.

[7] D. Cassioli, M. Z. Win, F. Vatalaro, and A. F. Molisch, "Performance of low-complexity Rake reception in a realistic UWB channel," in Proceedings of the IEEE International Conference on Communications (ICC '02), vol. 2, pp. 763-767, May 2002.

[8] J. D. Choi and W. E. Stark, "Performance of ultra-wideband communications with suboptimal receivers in multipath channels," IEEE Journal on Selected Areas in Communications, vol. 20, no. 9, pp. 1754-1766, 2002.

[9] M. Z. Win and R. A. Scholtz, "On the energy capture of ultrawide bandwidth signals in dense multipath environments," IEEE Communications Letters, vol. 2, no. 9, pp. 245-247, 1998.

[10] V. Lottici, A. D'Andrea, and U. Mengali, "Channel estimation for ultra-wideband communications," IEEE Journal on Selected Areas in Communications, vol. 20, no. 9, pp. 1638-1645, 2002.

[11] R. Hoctor and H. Tomlinson, "Delay-hopped transmittedreference RF communications," in Proceedings of the IEEE Conference on Ultra Wideband Systems and Technologies (UWBST '02), pp. 265-269, May 2002.

[12] Y.-L. Chao and R. A. Scholtz, "Optimal and suboptimal receivers for ultra-wideband transmitted reference systems," in Proceedings of the IEEE Global Telecommunications Conference (GLOBECOM '03), pp. 759-763, December 2003.

[13] T. Q. S. Quek and M. Z. Win, "Analysis of UWB transmittedreference communication systems in dense multipath channels," IEEE Journal on Selected Areas in Communications, vol. 23, no. 9, pp. 1863-1874, 2005.

[14] L. Yang and G. B. Giannakis, "Optimal pilot waveform assisted modulation for ultrawideband communications," IEEE Transactions on Wireless Communications, vol. 3, no. 4, pp. 12361249, 2004.

[15] S. Zhao, H. Liu, and Z. Tian, "Decision directed autocorrelation receivers for pulsed ultra-wideband systems," IEEE Transactions on Wireless Communications, vol. 5, no. 8, pp. 2175-2184, 2006.

[16] N. Guo and R. C. Qiu, "Improved autocorrelation demodulation receivers based on multiple-symbol detection for UWB communications," IEEE Transactions on Wireless Communications, vol. 5, no. 8, pp. 2026-2031, 2006.

[17] V. Lottici and Z. Tian, "Multiple symbol differential detection for UWB communications," IEEE Transactions on Wireless Communications, vol. 7, no. 5, pp. 1656-1666, 2008.

[18] J. Jaldén and B. Ottersten, "On the complexity of sphere decoding in digital communications," IEEE Transactions on Signal Processing, vol. 53, no. 4, pp. 1474-1484, 2005.

[19] S. Gezici, H. Kobayashi, H. V. Poor, and A. F. Molisch, "Performance evaluation of impulse radio UWB systems with pulse-based polarity randomization," IEEE Transactions on Signal Processing, vol. 53, no. 7, pp. 2537-2549, 2005.

[20] A. A. M. Saleh and R. A. Valenzuela, "A statistical model for indoor multipath propagation," IEEE Journal on Selected Areas in Communications, vol. 5, no. 2, pp. 128-137, 1987. 